\title{
Inelastic Scattering of Synchrotron Radiation from Electrons and Nuclei for Lattice Dynamics Studies
}

\author{
E. E. Alp, W. Sturhahn, H. Sinn, T. Toellner, M. Hu, J. Sutter, and A. Alatas \\ Advanced Photon Source, Argonne National Laboratory, * Argonne, IL 60439, USA
}

November 1999

The submitted manuscript has been created by
the University of Chicago as Operator of
Argonne National Laboratory ("Argonne") under
Contract No. W-31-109-ENG-38 with the U.S.
Department of Energy. The U.S. Government
retains for itself, and others acting on its behalf,
a paid-up, nonexclusive, irrevocable worldwide
license in said article to reproduce, prepare
derivative works, distribute copies to the public,
and perform publicly and display publicly, by
or on behalf of the Government.

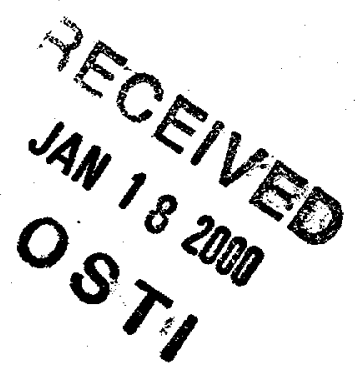

Invited paper presented at the $18^{\text {th }}$ International Conference on X-ray and Inner-Shell Processes, Chicago, IL, August 23-27, 1999; to be published in the Proceedings, eds. D. S. Gemmell, E. P. Kanter, and L. Young.

\footnotetext{
*This work is supported by the U.S. Department of Energy, Basic Energy Sciences, Office of Science, under contract \#W-31-109-ENG-38.
} 


\section{DISCLAIMER}

This report was prepared as an account of work sponsored by an agency of the United States Government. Neither the United States Government nor any agency thereof, nor any of their employees, make any warranty, express or implied, or assumes any legal liability or responsibility for the accuracy, completeness, or usefulness of any information, apparatus, product, or process disclosed, or represents that its use would not infringe privately owned rights. Reference herein to any specific commercial product, process, or service by trade name, trademark, manufacturer, or otherwise does not necessarily constitute or imply its endorsement, recommendation, or favoring by the United States Government or any agency thereof. The views and opinions of authors expressed herein do not necessarily state or reflect those of the United States Government or any agency thereof. 


\section{DISCLAIMER}

Portions of this document may be illegible in electronic image products. Images are produced from the best available original document. 


\title{
Inelastic Scattering of Synchrotron Radiation from Electrons and Nuclei for Lattice Dynamics Studies
}

\author{
E. E. Alp, W. Sturhahn, H. Sinn, T. Toellner, M. Hu, J. Sutter, \\ and A. Alatas
}

Advanced Photon Source, Argonne National Laboratory, Argonne, Illinois 60540

\begin{abstract}
The inelastic scattering of $x$-rays, one of the the first applications of $x$-rays to the field of condensed matter physics, has been rejuvenated in the last decade. The availability of synchrotron radiation from wiggler and undulator sources combined with advances in monochromatization of the incident beam and analysis of the scattered beam with $\mathrm{meV}$ resolution led to the measurement of phonon dispersion relations. In addition, the use of Mossbauer nuclei as scatterer and analyzers has led to the discovery of the inelastic nuclear resonant scattering technique. This new method allows extraction of partial phonon density of states from amorphous materials, thin films, multilayers and interfaces, and liquids.
\end{abstract}

\section{INTRODUCTION}

The early attempts of Debye [1] and the first determination of phonon dispersion relations by thermal diffuse scattering in $\mathrm{Al}$ [2] demonstrated the potential of $\mathrm{x}$-rays for vibrational studies. However, there was a period of low activity in the 1960 s and 70s due to lack of efficient analyzers with energy resolution commensurate with excitation energies, and not surprisingly, coincident with the development of inelastic neutron scattering [3].

The discovery of the Mössbauer effect [4] and the ensuing excitement over the possibility to use extremely monochromatic radioactive sources for lattice dynamics studies $[5,6]$ were dampened with the realization that both the range of energy tunability, and the bandwidth of the radiation were too small. However, the LambMössbauer factor, or recoiless fraction of absorption and emission of $\mathrm{x}$-rays, and the second order Doppler shift obtained from Mössbauer spectra proved to be useful for lattice dynamics studies. Similarly, element-specific Debye-Waller factors obtained from EXAFS data were used to understand lattice dynamics in addition to the more traditional techniques of infrared and Raman spectroscopy. 
The use of synchrotron radiation and the subsequent improvements in the source brilliance via the introduction of wigglers, coupled with clever use of crystal optics [7], led the way for a revival of inelastic x-ray scattering, which resulted in the first successful demonstration of phonon dispersion relation in Be [8]. Similarly, synchrotron radiation was used to observe coherent nuclear Bragg diffraction in $\mathrm{Y}_{3}{ }^{57} \mathrm{Fe}_{5} \mathrm{O}_{12}$ [9], and a decade later inelastic incoherent nuclear resonant scattering was realized [10] to extract phonon density of states [11].

\section{METHODOLOGY}

$\mathrm{X}$-rays are a suitable probe for a variety of excitations in condensed matter because of their ability to penetrate, their wavelength being comparable to interatomic distances, and the possibility to determine the exact amount of energy and momentum transfer during the scattering process. In a typical $x$-ray scattering experiment, photons with energy $E_{i}$ with a wavevector $\mathbf{k}_{i}$ and polarization $\mathbf{e}_{i}$ are incident onto a sample. The measurement then involves determination of the final energy $E_{\mathrm{f}}$, scattered into a new direction, $\mathbf{k}_{\mathfrak{f}}$, and polarization state, $\mathbf{e}_{\mathrm{f}}$. Since the energy analysis requires a certain solid angle to be intercepted, the scattering cross section is defined as functions of both frequency, $\omega$, and solid angle, $\Omega$ :

$$
\frac{d^{2} \sigma}{d \Omega d \omega}=\frac{d \sigma}{d \Omega} S(\mathbf{Q}, \omega)
$$

where $\mathbf{Q}=\mathbf{k}_{\mathrm{f}}-\mathbf{k}_{\mathrm{i}}$ is the momentum, and $\hbar \omega=E_{f}-E_{i}$ is the energy transfer.

The first term, describing the coherent coupling of electrons, is the Thomson cross section, $d \sigma / d \Omega=r_{0}^{2}\left(\mathbf{e}_{i} \cdot \mathbf{e}_{f}\right)^{2} \omega_{f} / \omega_{i}$, where $r_{0}$ is the classical electron radius. The second term, which describes the overall scattering strength of the probed volume, will depend on the dynamics of the particles in this volume. It is expressed in terms of the correlation of the phase of the scattering amplitude:

$$
S(\mathbf{Q}, \omega)=\sum_{i f} g_{i}\left|\left\langle i \sum_{j} e^{i Q r_{j}} \mid f\right\rangle\right|^{2} . \delta\left(\omega-\omega_{f}-\omega_{i}\right)
$$

where summation over $j$ is performed over all electrons, and $g_{i}$ is a weight factor for the initial states. This can also be written as a time Fourier transform of the density correlation function:

$$
S(\mathbf{Q}, \omega)=\frac{1}{2 \pi} \int d t\left(e^{-i \omega r} \sum_{i} g_{i} \mid\left(i\left|\sum_{j, l} e^{-i Q r_{j}(0)} e^{i Q r_{i}(t)}\right| i\right\rangle\right)
$$

For coherent elastic scattering, where there is no energy exchange, the cross section is reduced to $S(\mathbf{Q})=\left|\sum_{j} e^{i \mathbf{Q} \cdot \mathbf{r}_{i}}\right|^{2}$. The sum will vanish, unless $\mathbf{Q} \cdot \mathbf{r}_{j}$ is an integer multiple of $2 \pi$ for all sites. It can be shown that this condition is realized under Bragg 
diffraction, when the scattering vector $\mathbf{Q}$ is equal to the reciprocal lattice vector $\mathbf{G}$. Thus $S(Q)$ is a measure of the static structure factor.

Coherent inelastic scattering, on the other hand, will involve exchange of energy, in addition to momentum. A coherent scattering experiment in which the removal rate of photon (or neutron) is measured as a function of energy and momentum transfer yields information about the frequency and spatial extent of the phonons. We can describe phonons as quantized lattice vibrations of ion cores around their equilibrium positions. Coherent elastic or inelastic scattering of $x$-rays (neutrons) measure static or dynamic structure factors by scattering from core and outer electrons (nuclei). In the adiabatic approximation, the electrons and the nuclei move together, and neutron scattering experiments should yield the same result as $\mathrm{x}$-ray scattering experiments. However, for metals, the conduction electron gas need not behave according to the adiabatic approximation [12]. The only experimental evidence so far for the breakdown of the Born-Oppenheimer approximation is obtained from $\mathrm{x}$-ray diffraction measurements [13]. The difference in charge distribution in $\mathrm{LiH}$ and its $\mathrm{LiD}$ derivative is attributed to a coupling between vibrations and electronic states. Inelastic $\mathrm{x}$-ray scattering may provide more direct evidence and thus presents a unique opportunity to study the behavior of classical and quantum liquids [14].

Inelastic x-ray scattering has been used to determine the temperature dependence of a transition from normal to fast sound in liquid water and to establish a coupling between propagating density fluctuations and dynamical structural rearrangements in the liquid [15]. Similarly, positive dispersion of sound velocity is observed in liquid lithium, which can be associated with viscoelastic shear relaxation [16].

Coherent inelastic $x$-ray (neutron) scattering yields information about the dynamic structure factor. When the inverse of the transferred momentum is comparable to the interatomic distance and the transferred energy can be analyzed with meV resolution, collective excitations of ion cores, i.e., phonons can be measured. By varying the momentum transfer, the dependence of phonon energy on momentum, $\omega(\mathrm{k})$, can be determined. The dispersion relations can then be analyzed to develop a force model, which ultimately leads to a more accurate description of the interatomic potential. When complete dispersion relations are available, a method known as Born-von Kármán analysis provides a connection between force constants and the dispersion relations. Phonon density of states, which is a key ingredient in making the connection to the classical thermodynamic quantities like specific heat and vibrational entropy, can be derived by sampling the dispersion curves [17].

When there is no crystalline order, as in the case of liquids or amorphous solids, incoherent inelastic neutron scattering technique may be used to measure phonon density of states directly. However, when the material is in the form of a thin film or a monolayer at an interface, the traditional techniques fail to yield information about the vibrational properties. The recent discovery of inelastic nuclear resonant scattering 
of synchrotron radiation addresses some of these aspects of lattice dynamics. This method directly measures the partial phonon density of states.

The inelastic nuclear resonant interaction cross section is related to the nuclear resonance cross-section and phonon excitation probability as follows:

$$
\begin{aligned}
& \sigma(E, \mathbf{k}) \approx \sigma_{0} \Gamma S^{*}(\omega, \mathbf{k}) \\
& \sigma_{0}=\frac{2 \pi}{1+\alpha}\left(\frac{\hbar c}{E_{0}}\right)^{2} \frac{2 I_{\varepsilon}+1}{2 I_{g}+1}
\end{aligned}
$$

Here $\sigma_{0}$ is the nuclear resonance cross section, $\alpha$ is the internal conversion coefficient, $E_{0}$ is the nuclear resonance energy, $I_{g}$ and $I_{e}$ are the total angular momentum quantum number for the ground and the excited state of the nucleus, and $\Gamma$ is the linewidth of the excited state. The function $S^{*}(\omega, \mathbf{k})$ is not the same as the dynamic structure factor given earlier. It can be considered as a probability density for phonon excitation. It is a function of incident energy and momentum. It can be shown that $S^{*}(\omega, \mathbf{k})$ is related to the pair auto-correlation function $G(r, t)$ via a space and time Fourier transform. A set of specific sum rules put forward by Lipkin provides a procedure to extract lattice dynamics information such as specific heat and average force constant [18]. The extraction of phonon density of states from the experimental data is described elsewhere $[11,19]$.

\section{EXPERIMENTAL IMPLEMENTATION}

The two methods mentioned above, coherent inelastic scattering, and incoherent nuclear resonant scattering of synchrotron radiation can be implemented in various ways. The set up used at the Advanced Photon Source 3-ID beamline combines the resources for both experiments, and they are schematically shown in Figure 1.

The coherent inelastic $x$-ray spectrometer described in Figure 2(a) utilizes an "in-line" tunable high energy monochromator as a source, and a curved crystal back-scattering $\mathrm{Si}$ crystal as an analyzer. The monochromator is a combination of Si (4 4 0)-(15 113 3) nested channel-cut crystals. The analyzer is a Si (18 6 0) diced crystal with a $6 \mathrm{~m}$ bending radius. The overall energy resolution is $2 \mathrm{meV}$, with a momentum transfer range up to $5 \AA^{-1}$. The energy of the incident photons is $21.657 \mathrm{keV}$. A typical phonon measurement obtained from single crystalline Be is shown in Figure 2.

The inelastic nuclear resonant spectrometer uses a similar "in-line" monochromator, consisting of either a "nested" channel-cut geometry or two-flat crystal geometry [20] to achieve meV resolution at the $14.4125 \mathrm{keV}$ nuclear resonance of ${ }^{57} \mathrm{Fe}$ or 23.880 $\mathrm{keV}$ nuclear resonance of ${ }^{119} \mathrm{Sn}$. The choice of these isotopes was mainly motivated by the long lifetime of intermediate states that are comparable to the duration between the electron bunches in the storage ring. This separation is typically of the order of 100 
nsec. When the incident radiation is tuned around the nuclear resonance energy, which has an energy width less than $0.1 \mu \mathrm{eV}$, the phonon creation or annihilation may subtract or supplement the incident photon energy to excite the nuclear resonance. This can be unambiguously detected by the delayed fluorescence signal after the main pulse. A typical data set is given in Figure 3 a for amorphous and crystalline $\mathrm{Fe}_{2} \mathrm{~Tb}$. The central peak corresponds to the nuclear resonance, and side peaks are due to phonon annihilation(left) and creation (right) processes. The logarithmic scale used helps to see a few unique features of this method: i) high signal-to-noise ratio allows the observation of two phonon processes. The peak at $+44 \mathrm{meV}$ for crystalline $\mathrm{Fe}_{2} \mathrm{~Tb}$ is a two-phonon contribution associated with the $+22 \mathrm{meV}$ peak. ii) The asymmetry between phonon creation (right) and phonon annihilation (left) is related to phonon occupation probability, $S(-\omega, \mathbf{k})=e^{-\hbar \omega / k T} S(\omega, \mathbf{k})$, and provides a detailed balance for a given temperature. iii) Signal-to-background ratio over 5 orders of magnitude is due to the way measurements are done at a pulsed synchrotron source with time discrimination. In Figure $4 \mathrm{~b}$ the derived Fe partial phonon density of states are shown for the amorphous and the corresponding crystalline phase. These type of studies have been extended to thin films [21], multilayers, and biological systems. The amount of material required is of the order of micrograms, and therefore, in combination with microfocusing techniques, these type of measurements have been extended to materials under high pressure in diamond-anvil cells [22].

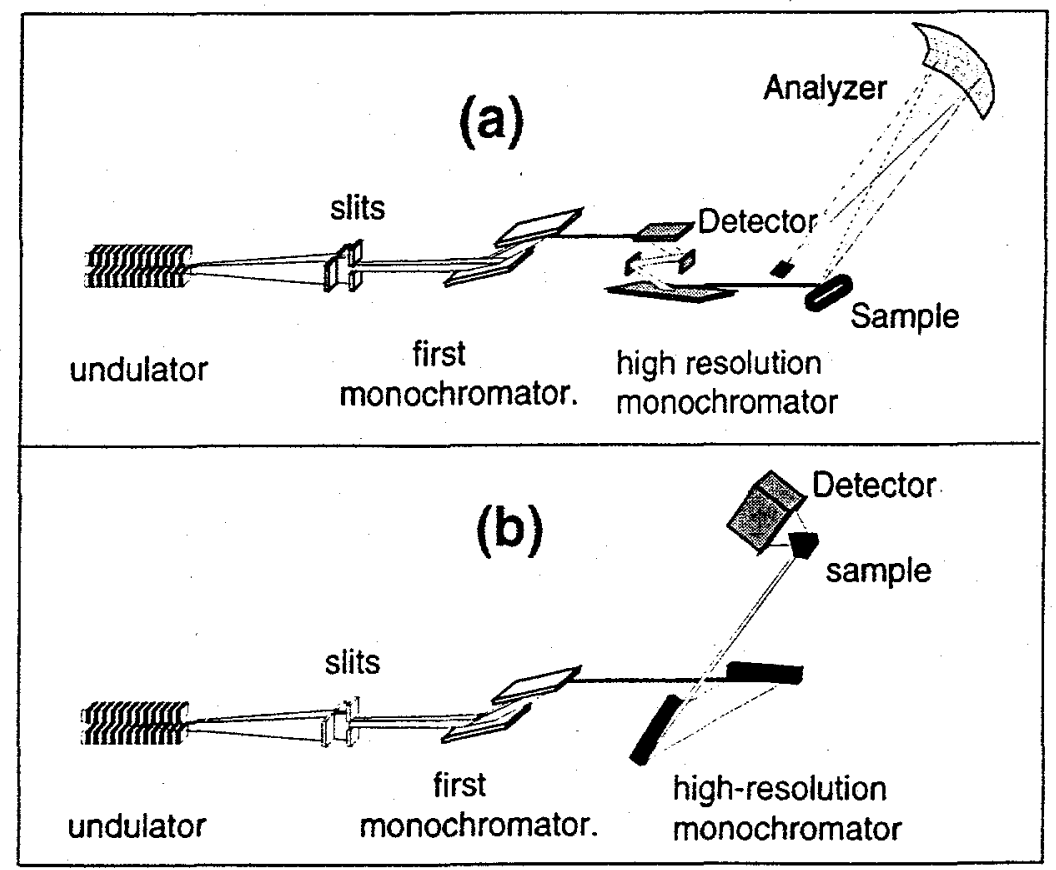

Figure 1 . The schematic realization of inelastic $x$-ray scattering experiments at the 3 ID beamline at the APS. (a) The inelastic $x$-ray spectrometer with an "in-line" monochromator, and a curved crystal analyzer with a bending radius of $6 \mathrm{~m}$. (b) The inelastic nuclear resonant spectrometer, with 2-flat crystal monochromator. The energy resolution is $0.6 \mathrm{meV}$ at $14.4 \mathrm{keV}^{57} \mathrm{Fe}$ resonance, and $0.5 \mathrm{meV}$ at $23.880 \mathrm{keV}$ ${ }^{119} \mathrm{Sn}$ resonance. 


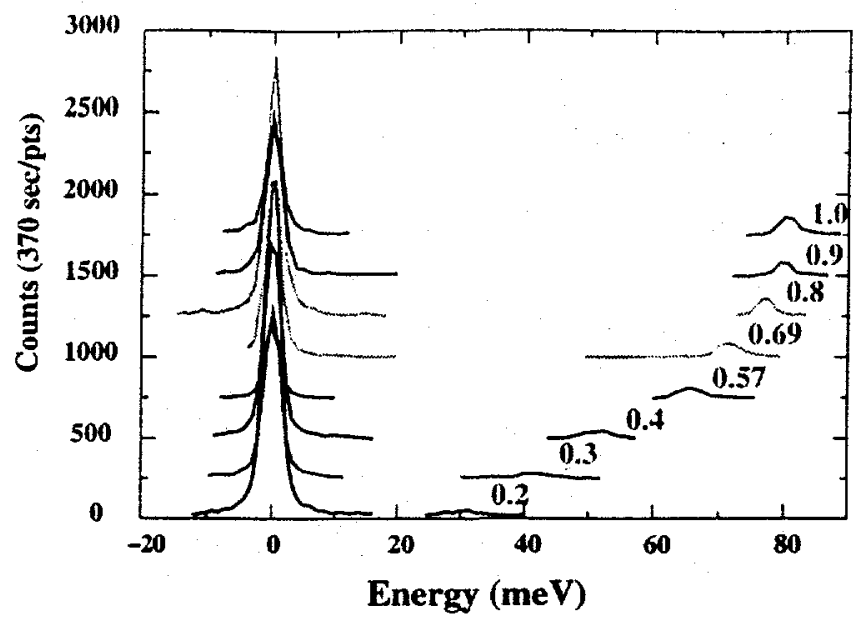

Figure 2. Phonon dispersion measurements in Be along $(00 \xi)$ direction for the longitudinal phonons for $\xi$ values indicated for each spectrum. The elastic peak is both due to the window material on the sample chamber, and impurities in beryllium.
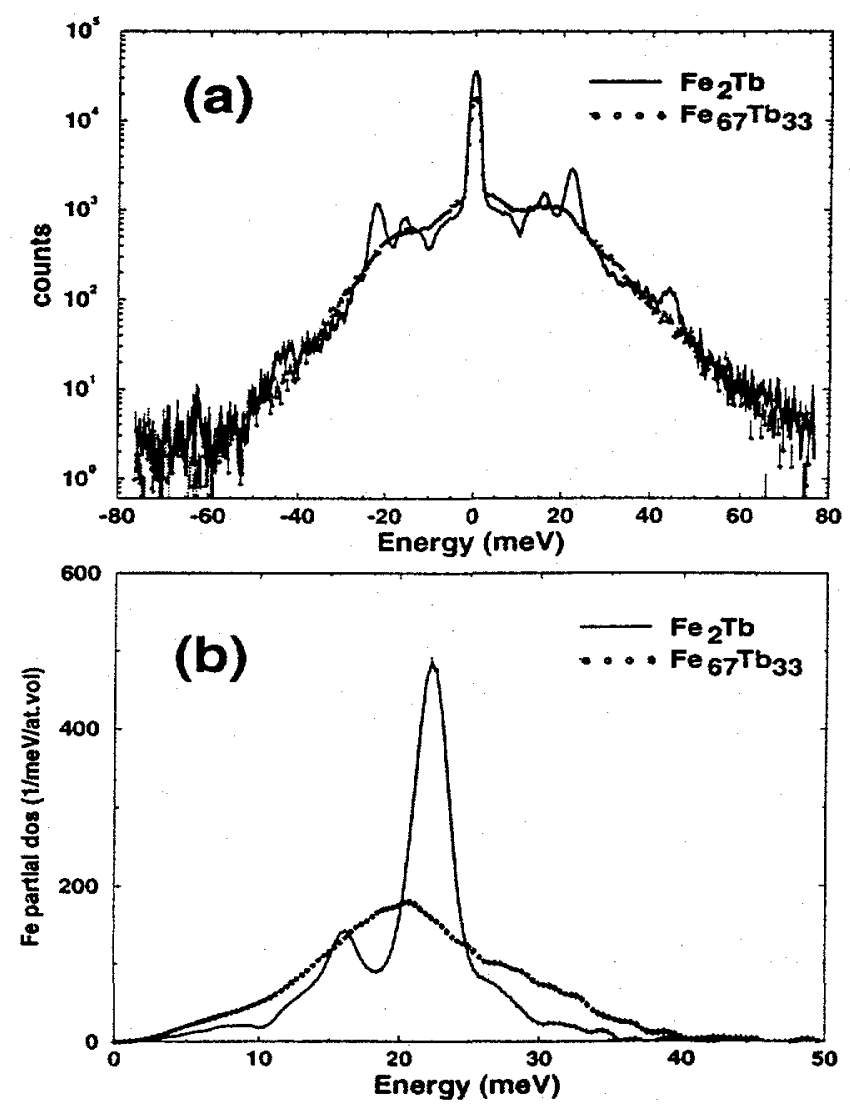

Figure 3. (a) The raw data measured around the ${ }^{57} \mathrm{Fe}$ resonance in $\mathrm{Fe}_{2} \mathrm{~Tb}$ alloy in crystalline and amorphous state, at room temperature. The samples were prepared in thin film form. The zero energy corresponds to the nuclear resonance energy of ${ }^{57} \mathrm{Fe}$ at $14.4125 \mathrm{keV}$. (b) The Fe partial density of states extracted from the data. 


\section{ACKNOWLEDGEMENTS}

This work is supported by US-DOE BES, under contract number W-31-109-ENG-38. We would like to thank Prof. W. Keune and T. Ruckert for providing the $\mathrm{Fe}_{2} \mathrm{~Tb}$ samples, and Prof. E. Burkel for the Be single crystal.

\section{REFERENCES}

1. P. Debye, Ann. Phys. 43 (1914) 49.

2. C. B. Walker, Phys. Rev. 103 (1956) 547.

3. B. N. Brockhouse, Inelastic Scattering of Neutrons in Solids and Liquids, IAEA, Vienna, (1961)

4. R. L. Mossbauer, Z. Physik, 151 (1958) 124.

5. V.M. Vischer, Annals of Physics, 9 (1960) 194.

6. K. S. Singwi, A. Sjolander, Phys. Rev. 120 (1960) 1093.

7. W. Graeff, G. Materlik, Nucl. Instrum. Meth., 195 (1982) 97.

8. B. Dorner, E. Burkel, Th. Illini, and J. Peisl, Z. Phys. B, Condensed Matter 69 (1987) 179.

9. E. Gerdau, R. Rüffer, H. Winkler, W. Tolksdorf, C. P. Kalges, J. P. Hannon, Phys. Rev. Lett. 54 (1985) 835.

10. M. Seto, Y. Yoda, S. Kikuta, X. W. Zhang, M. Ando, Phys. Rev. Lett. 74 (1995) 3828.

11. W. Sturhahn, T. S. Toellner, E. E. Alp, X. Zhang, M. Ando, S. Kikuta, M. Seto, C.W. Kimball, B. Dabrowski, Phys. Rev. Lett. 74 (1995) 3832.

12. M. Rasolt, Phys. Rev. B 31 (1984) 1615.

13. G. Vidal-Valat, J. -P. Vidal, Acta Cryst. A48 (1992) 46.

14. A.A. Louis, N. W. Aschcroft, J. Non. Cryst. Solids, 250-252 (1999) 9.

15. A. Cunsolo, G. Ruocco, F. Sette, C. Masciovecchio, A. Mermet, G. Monaco, M. Sampoli, R. Vernebi, Phys. Rev. Lett. 82 (1999) 775.

16. H. Sinn, F. Sette, U. Bergmann, Ch. Halcoissis, M. Krisch, R. Verbeni, and E. Burkel, Phys. Rev. Lett. 78 (1997) 1715.

17. G. Gilat, L, J, Raubenheimer, Phys. Rev. 144 (1965) 390.

18. H. J. Lipkin, Phys. Rev. B 52 (1995) 10073.

19. M. Hu, W. Sturhahn, T.S. Toellner, P. M. Hession, J. P. Sutter, E. E. Alp, Nucl. Instrum. Meth. A 428 (1999) 551.

20. T. S. Toellner, M. Y. Hu, W. Sturhahn, K. Quast, and E. E. Alp, Appl. Phys. Lett., 71 (1997) 2112.

21. W. Sturhahn, R. Röhlsberger, E. E. Alp, T. Ruckert, H. Schör, and W. Keune, J. Magnetism And Magnetic Materials, 198-199 (1999) 590.

22. H. K. Mao, W. Sturhahn, et al, (to be published, Argonne National Laboratory) 